\title{
Non-Vertical Cultural Transmission, Assortment, and the Evolution of Cooperation
}

\section{Dor Cohen ${ }^{1}$, Ohad Lewin-Epstein ${ }^{2}$, Marcus W. Feldman ${ }^{3}$, and Yoav Ram ${ }^{1,4,5, *}$}

4
${ }^{1}$ School of Computer Science, Interdisciplinary Center Herzliya, Herzliya, Israel

${ }^{2}$ School of Plant Sciences and Food Security, Faculty of Life Sciences, Tel Aviv University, Tel Aviv, Israel

${ }^{3}$ Department of Biology, Stanford University, Stanford, CA

${ }^{4}$ School of Zoology, Faculty of Life Sciences, Tel Aviv University, Tel Aviv, Israel

${ }^{5}$ Sagol School of Neuroscience, Tel Aviv University, Tel Aviv, Israel

*Corresponding author: yoav@yoavram.com

December 8, 2020

\begin{abstract}
Cultural evolution of cooperation under vertical and non-vertical cultural transmission is studied, and conditions are found for fixation and coexistence of cooperation and defection. The evolution of cooperation is facilitated by its horizontal transmission and by an association between social interactions and horizontal transmission. The effect of oblique transmission depends on the horizontal transmission bias. Stable polymorphism of cooperation and defection can occur, and when it does, reduced association between social interactions and horizontal transmission evolves, which leads to a decreased frequency of cooperation and lower population mean fitness. The deterministic conditions are compared to outcomes of stochastic simulations of structured populations. Parallels are drawn with Hamilton's rule incorporating assortment and effective relatedness.
\end{abstract}




\section{Introduction}

Cooperative behavior can reduce an individual's fitness and increase the fitness of its conspecifics or competitors [2]. Nevertheless, cooperative behavior appears to occur in many animals [5], including humans, primates [13], rats [24], birds [15, 27], and lizards [26]. Evolution of cooperative behavior has been an important focus of research in evolutionary theory since at least the 1930s [11, Appendix].

Since the work of Hamilton [12] and Axelrod \& Hamilton [2], theories for the evolution of cooperative and altruistic behaviors have been intertwined often under the rubric of kin selection. Kin selection theory posits that natural selection is more likely to favor cooperation between more closely related individuals. The importance of relatedness to the evolution of cooperation and altruism was demonstrated by Hamilton [12], who showed that an allele that determines cooperative behavior will increase in frequency if the reproductive cost to the actor that cooperates, $c$, is less than the benefit to the recipient, $b$, times

$$
c<b \cdot r
$$

where the relatedness coefficient $r$ measures the probability that an allele sampled from the cooperator is identical by descent to one at the same locus in the recipient.

Eshel \& Cavalli-Sforza [6] studied a related model for the evolution of cooperative behavior. Their model included assortative meeting, or non-random encounters, where a fraction $m$ of individuals in the population each interact specifically with an individual of the same phenotype, and a fraction $1-m$ interacts with a randomly chosen individual. Such assortative meeting may be due, for example, to population structure or active partner choice. In their model, cooperative behavior can evolve if [6, eq. 3.2]

$$
c<b \cdot m,
$$

where $b$ and $c$ are the benefit and cost of cooperation ${ }^{1}$.

The role of assortment in the evolution of altruism was emphasized by Fletcher \& Doebeli [9]. They

found that in a public-goods game, altruism will evolve if cooperative individuals experience more cooperation, on average, than defecting individuals, and "thus, the evolution of altruism requires (positive) assortment between focal cooperative players and cooperative acts in their interaction environment." With some change in parameters, this condition is summarized by [9, eq. 2.3]

$$
c<b \cdot\left(p_{C}-p_{D}\right),
$$

where $p_{C}$ is the probability that a cooperator receives help, and $p_{D}$ is the probability that a defector receives help. ${ }^{2}$ Bijma \& Aanen [3] obtained a result related to inequality 3 for other types of games.

Cooperative behavior can be subject to cultural transmission, which allows an individual to acquire attitudes or behavioral traits from other individuals in its social group through imitation, learning, or other modes of communication $[4,25]$. Feldman et al. [8] introduced the first model for the evolution of altruism by cultural transmission with kin selection and demonstrated that if the fidelity of cultural transmission of altruism is $\varphi$, then the condition for evolution of altruism in the case of sib-to-sib altruism is [8, Eq. 16]

$$
c<b \cdot \varphi-\frac{1-\varphi}{\varphi}
$$

\footnotetext{
${ }^{1}$ In an extended model, which allows an individual to encounter $N$ individuals before choosing a partner, the right hand side is multiplied by $E[N]$, the expected number of encounters [6, eq. 4.6].

${ }^{2}$ Inequality 3 generalizes inequalities 1 and 2 by substituting $p_{C}=r+p, p_{D}=p$ and $p_{C}=m+(1-m) p, p_{D}=(1-m) p$, respectively, where $p$ is the frequency of cooperators.
} 
In inequality 4, $\varphi$ takes the role of relatedness ( $r$ in inequality 1 ) or assortment ( $m$ in inequality 2$)$, but the effective benefit $b \cdot \varphi$ is reduced by $(1-\varphi) / \varphi$. This shows that under a cultural transmission, the condition for the evolutionary success of altruism entails a modification of Hamilton's rule (inequality 1).

66 Cultural transmission may be modeled as vertical, horizontal, or oblique: vertical transmission occurs between parents and offspring, horizontal transmission occurs between individuals from the same generation, and oblique transmission occurs to offspring from the generation to which their parents belong (i.e. from non-parental adults). Evolution under either of these transmission models can be be more rapid than under pure vertical transmission [4, 21, 23]. Both Woodcock [28] and Lewin-Epstein et al. [16] demonstrated that non-vertical transmission can help explain the evolution of cooperative behavior, the former using simulations with cultural transmission, the latter using a model where cooperation is mediated by host-associated microbes. Indeed, models in which microbes affect their host's behavior $[10,16,17]$ are mathematically similar to models of cultural transmission, and they also emphasize the role of non-vertical transmission [4].

Here, we study models for the cultural evolution of cooperation that include both vertical and nonvertical transmission. In our models behavioral changes are mediated by cultural transmission that can occur specifically during social interactions. For instance, there may be an association between the choice of partner for social interaction and the choice of partner for cultural transmission, or when an individual interacts with an individual of a different phenotype, exposure to the latter may lead the former to convert its phenotype. Our results demonstrate that cultural transmission, when associated with social interactions, can enhance the evolution of cooperation even when genetic transmission cannot, partly because it can facilitate the generation of assortment [9], and partly because it can diminish the effect of natural selection [23]. This further emphasizes that treatment of cooperation as a cultural trait, rather than a genetic one, can lead to a broader understanding of its evolutionary dynamics.

\section{Models}

88 Consider a large population whose members can be one of two phenotypes: $\phi=A$ for cooperators or $\phi=B$ for defectors. An offspring inherits its phenotype from its parent via vertical transmission with probability $v$ or from a random individual in the parental population via oblique transmission with probability $(1-v)$ (Figure 1a). Following Ram et al. [23], given that the parent's phenotype is $\phi$ and assuming uni-parental inheritance [29], the conditional probability that the phenotype $\phi^{\prime}$ of the offspring is $A$ is

$$
P\left(\phi^{\prime}=A \mid \phi\right)=\left\{\begin{array}{ll}
v+(1-v) p, & \text { if } \phi=A \\
(1-v) p, & \text { if } \phi=B
\end{array},\right.
$$

where $p=P(\phi=A)$ is the frequency of $A$ among all adults in the parental generation.

96 Not all adults become parents, and we denote the frequency of phenotype $A$ among parents by $\tilde{p}$. Therefore, the frequency $\hat{p}$ of phenotype $A$ among juveniles (after selection and vertical and oblique transmission) is

$$
\begin{aligned}
\hat{p}= & \tilde{p}[v+(1-v) p]+(1-\tilde{p})[(1-v) p]= \\
& v \tilde{p}+(1-v) p .
\end{aligned}
$$

100 Individuals are assumed to interact according to a prisoner's dilemma. Specifically, individuals interact in pairs; a cooperator suffers a fitness cost $0<c<1$, and its partner gains a fitness benefit 
102

104

$b$, where we assume $c<b$. Figure 1a shows the payoff matrix, i.e. the fitness of an individual with phenotype $\phi_{1}$ when interacting with a partner of phenotype $\phi_{2}$.

Social interactions occur randomly: two juvenile individuals with phenotype $A$ interact with probability $\hat{p}^{2}$, two juveniles with phenotype $B$ interact with probability $(1-\hat{p})^{2}$, and two juveniles with different phenotypes interact with probability $2 \hat{p}(1-\hat{p})$. Horizontal cultural transmission occurs between pairs of individuals from the same generation. It occurs between socially interacting partners with probability $\alpha$, or between a random pair with probability $1-\alpha$ (see Figure 1b). However, horizontal transmission is not always successful, as one partner may reject the other's phenotype. The probability of successful horizontal transmission of phenotypes $A$ and $B$ are $T_{A}$ and $T_{B}$, respectively (Table 1, Figure 1c). Then, the frequency $p^{\prime}$ of phenotype $A$ among adults in the next generation, after horizontal transmission, is

$$
\begin{aligned}
p^{\prime}= & \hat{p}^{2}\left[\alpha+(1-\alpha)\left(\hat{p}+(1-\hat{p})\left(1-T_{B}\right)\right)\right]+ \\
& \hat{p}(1-\hat{p})\left[\alpha\left(1-T_{B}\right)+(1-\alpha)\left(\hat{p}+(1-\hat{p})\left(1-T_{B}\right)\right)\right]+ \\
& (1-\hat{p}) \hat{p}\left[\alpha T_{A}+(1-\alpha) \hat{p} T_{A}\right]+ \\
& (1-\hat{p})^{2}\left[(1-\alpha) \hat{p} T_{A}\right],
\end{aligned}
$$

which simplifies to

$$
p^{\prime}=\hat{p}^{2}\left(T_{B}-T_{A}\right)+\hat{p}\left(1+T_{A}-T_{B}\right) .
$$

The frequency of $A$ among parents (i.e. after selection) follows a similar dynamic, but also includes the effect of natural selection, and is therefore

$$
\begin{aligned}
\bar{w} \tilde{p}^{\prime}= & \hat{p}^{2}(1+b-c)\left[\alpha+(1-\alpha)\left(\hat{p}+(1-\hat{p})\left(1-T_{B}\right)\right)\right]+ \\
& \hat{p}(1-\hat{p})(1-c)\left[\alpha\left(1-T_{B}\right)+(1-\alpha)\left(\hat{p}+(1-\hat{p})\left(1-T_{B}\right)\right)\right]+ \\
& (1-\hat{p}) \hat{p}(1+b)\left[\alpha T_{A}+(1-\alpha) \hat{p} T_{A}\right]+ \\
& (1-\hat{p})^{2}\left[(1-\alpha) \hat{p} T_{A}\right]
\end{aligned}
$$

where fitness values are taken from Figure 1a and Table 1, and the population mean fitness is $\bar{w}=1+\hat{p}(b-c)$. Eq. 9 can be simplified to

$$
\begin{aligned}
\bar{w} \tilde{p}^{\prime}= & \left.\hat{p}^{2}(1+b-c)\left[1-(1-\hat{p})(1-\alpha) T_{B}\right)\right]+ \\
& \hat{p}(1-\hat{p})(1-c)\left[\hat{p}(1-\alpha) T_{B}+1-T_{B}\right]+ \\
& (1-\hat{p}) \hat{p}(1+b)[\hat{p}(1-\alpha)+\alpha] T_{A}+ \\
& (1-\hat{p})^{2} \hat{p}(1-\alpha) T_{A} .
\end{aligned}
$$

Starting from Eq. 6 with $\hat{p}^{\prime}=v \tilde{p}^{\prime}+(1-v) p^{\prime}$, we substitute $p^{\prime}$ from Eq. 8 and $\tilde{p}^{\prime}$ from Eq. 10 and obtain

$$
\begin{aligned}
\hat{p}^{\prime}= & \left.\frac{v}{\bar{w}}\left[\hat{p}^{2}(1+b-c)\left(1-(1-\hat{p})(1-\alpha) T_{B}\right)\right)\right]+ \\
& \frac{v}{\bar{w}}\left[\hat{p}(1-\hat{p})(1-c)\left(\hat{p}(1-\alpha) T_{B}+1-T_{B}\right)\right]+ \\
& \frac{v}{\bar{w}}\left[\hat{p}(1-\hat{p})(1+b)(\hat{p}(1-\alpha)+\alpha) T_{A}\right]+ \\
& \frac{v}{\bar{w}}(1-\hat{p})^{2} \hat{p}(1-\alpha) T_{A}+ \\
& (1-v) \hat{p}^{2}\left(T_{B}-T_{A}\right)+ \\
& (1-v) \hat{p}\left(1+T_{A}-T_{B}\right) .
\end{aligned}
$$

Table 2 lists the model variables and parameters. 
126

\section{Results}

We determine the equilibria of the model in Eq. 11, namely, solutions of $\hat{p}^{\prime}=\hat{p}$, and analyze their local stability. We then analyze the evolution of a modifier of interaction-transmission association, $\alpha$. Finally, we compare derived conditions to outcomes of stochastic simulations with a structured population.

\section{Evolution of cooperation}

The fixed points (equilibria) of the recursion (Eq. 11) are $\hat{p}=0, \hat{p}=1$, and (see Eq. B5)

$$
\hat{p}^{*}=\frac{\alpha b v T_{A}-c v\left(1-T_{B}\right)+\left(T_{A}-T_{B}\right)}{[c(1-v)-b(1-\alpha v)]\left(T_{A}-T_{B}\right)} .
$$

Define the following cost thresholds, $\gamma_{1}$ and $\gamma_{2}$, and the vertical transmission threshold, $\hat{v}$,

$$
\gamma_{1}=\frac{b v \alpha T_{A}+\left(T_{A}-T_{B}\right)}{v\left(1-T_{B}\right)}, \quad \gamma_{2}=\frac{b v \alpha T_{B}+(1+b)\left(T_{A}-T_{B}\right)}{v\left(1-T_{B}\right)+(1-v)\left(T_{A}-T_{B}\right)}, \quad \hat{v}=\frac{T_{B}-T_{A}}{1-T_{A}} .
$$

Then we have the following result.

Result 1 (Equilibria and stability). With vertical, horizontal, and oblique transmission, the cultural evolution of a cooperation follows one of the following scenarios in terms of the cost thresholds $\gamma_{1}$ and $\gamma_{2}$ and the vertical transmission threshold $\hat{v}$ (Eq. 13) :

1. Fixation of cooperation: if (i) $T_{A} \geq T_{B}$ and $c<\gamma_{1}$; or if (ii) $T_{A}<T_{B}$ and $v>\hat{v}$ and $c<\gamma_{2}$.

2. Fixation of defection: if (iii) $T_{A} \geq T_{B}$ and $\gamma_{2}<c$; or if (iv) $T_{A}<T_{B}$ and $\gamma_{1}<c$.

3. Stable polymorphism: if (v) $T_{A}<T_{B}$ and $v<\hat{v}$ and $c<\gamma_{1}$; or if (vi) $T_{A}<T_{B}$ and $v>\hat{v}$ and $\gamma_{2}<c<\gamma_{1}$.

4. Unstable polymorphism: if (vii) $T_{A}>T_{B}$ and $\gamma_{1}<c<\gamma_{2}$.

These conditions are illustrated in Figures 2a, 2b, 3a, and 3b, and the full analysis is in Appendix B.

Much of the literature on evolution of cooperation focuses on conditions for an initially rare cooperative phenotype to invade a population of defectors. The following remarks address this condition.

Remark 1 (Condition on $c$ for cooperation to increase when rare, i.e., $\hat{p}^{\prime}>p$ near $p=0$ ). If the initial frequency of cooperation is very close to zero, then its frequency will increase if the cost of cooperation is low enough,

$$
c<\gamma_{1}=\frac{b v \alpha T_{A}+\left(T_{A}-T_{B}\right)}{v\left(1-T_{B}\right)} .
$$

This unites the conditions for fixation of cooperation and for stable polymorphism, both of which entail instability of the state where defection is fixed, $\hat{p}=0$.

Importantly, increasing interaction-transmission association $\alpha$ increases the cost threshold $\left(\partial \gamma_{1} / \partial \alpha>\right.$ 0 ), making it easier for cooperation to increase in frequency when initially rare. Similarly, increasing the horizontal transmission of cooperation, $T_{A}$, increases the threshold $\left(\partial \gamma_{1} / \partial T_{A}>0\right)$, facilitating the evolution of cooperation ((Figure $3 \mathrm{a}$ and $3 \mathrm{~b})$. However, increasing the horizontal transmission of 
defection, $T_{B}$, can increase or decrease the cost threshold, but it increases the cost threshold when the threshold is already above one $\left(c<1<\gamma_{1}\right)$ : $\partial \gamma_{1} / \partial T_{B}$ is positive when $T_{A}>\frac{1}{1+\alpha b v}$, which gives $\gamma_{1}>1 / v$. Therefore, increasing $T_{B}$ decreases the cost threshold and limits the evolution of cooperation, but only if $T_{A}<\frac{1}{1+\alpha b v}$.

Increasing the vertical transmission rate, $v$, can either increase or decrease the cost threshold, depending on the horizontal transmission bias, $T_{A}-T_{B}$, because $\operatorname{sign}\left(\partial \gamma_{1} / \partial v\right)=-\operatorname{sign}\left(T_{A}-T_{B}\right)$. When $T_{A}<T_{B}$ we have $\partial \gamma_{1} / \partial v>0$, and as the vertical transmission rate increases, the cost threshold increases, making it easier for cooperation to increase when rare (Figure $2 \mathrm{~b}$ ). In contrast, when $T_{A}>T_{B}$ we get $\partial \gamma_{1} / \partial v<0$, and therefore as the vertical transmission rate increases, the cost threshold decreases, making it harder for cooperation to increase when rare (Figure 2a).

In general, this condition cannot be formulated in the form of Hamilton's rule due to the bias in horizontal transmission, represented by $T_{A}-T_{B}$. When there is no horizontal transmission bias, $T_{A}=T_{B}$, the following applies.

From Result 1 and inequality 14, if horizontal transmission is unbiased, $T=T_{A}=T_{B}$, then cooperation will take over the population from any initial frequency if the cost is low enough,

$$
c<b \cdot \frac{\alpha T}{1-T}
$$

and regardless of the vertical transmission rate, $v$. This condition can be interpreted as a version of Hamilton's rule $(c<b \cdot r$, inequality 1$)$ or as a version of inequality 3 , where $\alpha T /(1-T)$ can be regarded as the effective relatedness or effective assortment, respectively. Note that the right-hand side of inequality 15 equals $\gamma_{1}$ when $T=T_{A}=T_{B}$.

From inequality 14, without interaction-transmission association $(\alpha=0)$, cooperation will increase when it is rare if there is horizontal transmission bias for cooperation, $T_{A}>T_{B}$, and

$$
c<\frac{T_{A}-T_{B}}{v\left(1-T_{B}\right)} .
$$

Figure 3a illustrates this condition (for $v=1$ ), which is obtained by setting $\alpha=0$ in inequality 14 . Importantly, the benefit of cooperation, $b$, does not affect the evolution of cooperation in the absence of interaction-transmission association, and the outcome is determined only by cultural transmission. Further, inequality 14 shows that with perfect interaction-transmission association $(\alpha=1)$, cooperation will increase when rare if

$$
c<\frac{b v T_{A}+\left(T_{A}-T_{B}\right)}{v\left(1-T_{B}\right)} .
$$

In the absence of oblique transmission, $v=1$, the only equilibria are the fixation states, $\tilde{p}=0$ and $\tilde{p}=1$, and cooperation will evolve from any initial frequency (i.e., $\tilde{p}^{\prime}>\tilde{p}$ ) if inequality 17 applies (Figure 3). This is similar to case of microbe-induced cooperation studied by Lewin-Epstein et al. [16]; therefore when $v=1$, this remark is equivalent to their eq. 1 .

$$
a_{1}=\frac{c \cdot v\left(1-T_{A}\right)-\left(T_{A}-T_{B}\right)(1+b-c)}{b \cdot v \cdot T_{B}}, \quad a_{2}=\frac{c \cdot v\left(1-T_{B}\right)-\left(T_{A}-T_{B}\right)}{b \cdot v \cdot T_{A}} .
$$

Remark 2 (Condition on interaction-transmission association $\alpha$ for cooperation to increase when it is rare). Cooperation will increase when rare if interaction-transmission association is high enough, specifically if $a_{2}<\alpha$. 
200

202

204

208

210

212

Next, we examine the roles of vertical and oblique transmission in the evolution of cooperation.

Fixation of cooperation is possible only if the vertical transmission rate is high enough,

$$
v>\hat{v}=\frac{T_{B}-T_{A}}{1-T_{A}} .
$$

216

which can also be written as

$$
\frac{c\left(1-T_{B}\right)-\left(T_{A}-T_{B}\right)}{b T_{A}}<\alpha .
$$

\section{Evolution of interaction-transmission association}

We now focus on the evolution of interaction-transmission association under perfect vertical transmission, $v=1$, assuming that the population is initially at a stable polymorphism of the two phenotypes, 


\section{Population structure} (Figure 4).

cooperation $A$ and defection $B$, where the frequency of $A$ among juveniles is $\hat{p}^{*}$ (Eq. 12). Note that for a stable polymorphism, there must be horizontal bias for defection, $T_{A}<T_{B}$, and an intermediate cost of cooperation, $\gamma_{2}<c<\gamma_{1}$ (Eq. 13), see Figure 3b. The equilibrium population mean fitness is $\bar{w}^{*}=1+\hat{p}^{*}(b-c)$, which is increasing in $\hat{p}^{*}$, and $\hat{p}^{*}$ is increasing in $\alpha$ (Appendix C). Therefore, if interaction-transmission association increases, the population mean fitness also increases. But can this population-level advantage lead to the evolution of interaction-transmission association?

To answer this question, we extend our model to include a "modifier locus" [7, 18, 19, 20] that determines interaction-transmission association, but has no direct effect on fitness. The modifier locus has two alleles, $M$ and $m$, which induce interaction-transmission associations $\alpha_{1}$ and $\alpha_{2}$, respectively. Suppose that the population has evolved to a stable equilibrium $\hat{p}^{*}$ when only allele $M$ is present. We study the local stability of this equilibrium to invasion by the modifier allele $m$; this is called "external stability" $[1,20]$ and obtain the following result.

Result 2 (Reduction principle for interaction-transmission association). From a stable polymorphism between cooperation and defection, a modifier allele can successfully invade the population if it decreases the interaction-transmission association $\alpha$.

The full analysis is in Appendix D. Note that this reduction principle entails that successful invasions will reduce the frequency of cooperation, as well as the population mean fitness (Figure 4). Furthermore, if we assume that modifier alleles with decreased interaction-transmission association appear and invade the population from time to time, then interaction-transmission association will continue to decrease, further reducing the frequency of cooperation and the population mean fitness. This evolution will proceed as long as there is a stable polymorphism, that is, as long as $a_{2}<\alpha<a_{1}$ (Remark 2, Figure 3c). Thus, we can expect interaction-transmission association to eventually approach $a_{2}$, the frequency of cooperation to fall to zero, and the population mean fitness to decrease to one

Interaction-transmission association may also emerge from population structure. Consider a population colonizing a two-dimensional grid of size 100-by-100, where each site is inhabited by one individual, similarly to the model of Lewin-Epstein \& Hadany [17]. Each individual is characterized by its phenotype: either cooperator, $A$, or defector, $B$. Initially, each site in the grid is randomly colonized by either a cooperator or a defector, with equal probability. In each generation, half of the individuals are randomly chosen to "initiate" interactions, and these initiators interact with a random neighbor (i.e. individual in a neighboring site) in a prisoners' dilemma game (Figure 1a) and a random neighbor (with replacement) for horizontal cultural transmission (Figure 1b). The expected number of each of these interactions per individual per generation is one. The effective interaction-transmission association $\alpha$ in this model is the probability that the same neighbor is picked for both interactions, or $\alpha=1 / M$, where $M$ is the number of neighbors. On an infinite grid, $M=8$, but on a finite grid $M$ can be lower in edge neighborhoods close to the grid border. As before, $T_{A}$ and $T_{B}$ are the probabilities of successful horizontal transmission of phenotypes $A$ and $B$, respectively.

The order of the interactions across the grid at each generation is random. After all interactions take place, an individual's fitness is determined by $w=1+b \cdot n_{b}-c \cdot n_{c}$, where $n_{b}$ is the number of interactions that individual had with cooperative neighbors, and $n_{c}$ is the number of interactions in which that individual cooperated (note that the phenotype may change between consecutive interactions due to horizontal transmission). Then, a new generation is produced, and the sites can be settled by offspring of any parent, not just the neighboring parents. Selection is global, rather than local, in accordance with our deterministic model: The parent is randomly drawn with probability proportional to its 
288

290

292

294

296

298

300

302

304

306

308

310

312

314

316

318

320

322

fitness, divided by the sum of the fitness values of all potential parents. Offspring are assumed to have the same phenotype as their parents (i.e. $v=1$ ).

The outcomes of stochastic simulations with such a structured population are shown in Figure 5, which demonstrates that the highest cost of cooperation $c$ that permits the evolution of cooperation agrees with the conditions derived above for our model without population structure or stochasticity. An example of stochastic stable polymorphism is shown in Figure 5c. Changing the simulation so that selection is local (i.e., sites can only be settled by offspring of neighboring parents) had only a minor effect on the agreement with the derived conditions (Figure S1).

These comparisons between the deterministic unstructured model and the stochastic structured model show that the conditions derived for the deterministic model can be useful for predicting the dynamics under complex scenarios. Moreover, this structured population model demonstrates that our parameter for interaction-transmission association, $\alpha$, can represent local interactions between individuals.

\section{Discussion}

Under a combination of vertical, oblique, and horizontal transmission with payoffs in the form of a prisoner's dilemma game, cooperation or defection can either fix or coexist, depending on the relationship between the cost and benefit of cooperation, the horizontal transmission bias, and the association between social interaction and horizontal transmission (Result 1, Figures 2 and 3). Importantly, cooperation can increase when initially rare (i.e. invade a population of defectors) if and only if, rewriting inequality 14 ,

$$
c \cdot v\left(1-T_{B}\right)<b \cdot v \alpha T_{A}+\left(T_{A}-T_{B}\right),
$$

namely, the effective cost of cooperation (left-hand side) is smaller then the effective benefit plus the horizontal transmission bias (right-hand side). This condition cannot be formulated in the form of Hamilton's rule, $c<b \cdot r$, due to the effect of biased horizontal transmission, represented by $\left(T_{A}-T_{B}\right)$. Remarkably, a polymorphism of cooperation and defection can be stable if horizontal transmission is biased in favor of defection $\left(T_{A}<T_{B}\right)$ and both the cost of cooperation and interaction-transmission association are intermediate (yellow areas in Figures 2 and 3 ).

We find that stronger interaction-transmission association $\alpha$ leads to evolution of higher frequency of cooperation and increased population mean fitness. Nevertheless, when cooperation and defection coexist, interaction-transmission association is expected to be reduced by natural selection, leading to extinction of cooperation and decreased population mean fitness (Result 2, Figure 4). Without interaction-transmission association, the benefit of cooperation cannot facilitate its evolution, and cooperation can only succeed if horizontal transmission is biased in its favor.

Indeed, in our model, horizontal transmission plays a major role in the evolution of cooperation: increasing the transmission of cooperation, $T_{A}$, or decreasing the transmission of defection, $T_{B}$, facilitates the evolution of cooperation. However, the effect of oblique transmission is more complicated. When there is horizontal transmission bias in favor of cooperation, $T_{A}>T_{B}$, increasing the rate of oblique transmission, $1-v$, will facilitate the evolution of cooperation. In contrast, when the bias is in favor of defection, $T_{A}<T_{B}$, higher rates of vertical transmission, $v$, are advantageous for cooperation, and the rate of vertical transmission must be high enough $(v>\hat{v})$ for cooperation to fix in the population.

The conditions derived from our deterministic model provide a good approximation to outcomes of simulations of a complex stochastic model with population structure in which individuals can only interact with and transmit to their neighbors. In these structured populations interaction-transmission association arises due to both social interactions and horizontal cultural transmission being local (Figure 5). 
332 Feldman et al. [8] studied the dynamics of an altruistic phenotype with vertical cultural transmission and a gene that modifies the transmission of the phenotype. Their results are very sensitive to

334 this genetic modification: without it, the conditions for invasion of the altruistic phenotype reduce to Hamilton's rule. Further work is needed to incorporate such genetic modification of cultural

336 transmission into our model. Woodcock [28] stressed the significance of non-vertical transmission for the evolution of cooperation and carried out simulations with prisoner's dilemma payoffs but without

338 horizontal transmission or interaction-transmission association $(\alpha=0)$. Nevertheless, his results demonstrated that it is possible to sustain altruistic behavior via cultural transmission for a substantial

340 length of time. He further hypothesized that horizontal transmission can play an important role in the evolution of cooperation, and our results provide strong evidence for this hypothesis.

342 To understand the role of horizontal transmission, we first review the role of assortment. Eshel \& Cavalli-Sforza [6] showed that altruism can evolve when the tendency for assortative meeting, i.e.,

344 for individuals to interact with others of their own phenotype, is strong enough. Fletcher \& Doebeli [9] further argued that a general explanation for the evolution of altruism is given by assortment: the

346 correlation between individuals that carry an altruistic trait and the amount of altruistic behavior in their interaction group (see also Bijma \& Aanen [3]). They suggested that to explain the evolution of

348 altruism, we should seek mechanisms that generate assortment, such as population structure, repeated interactions, and individual recognition. Our results highlight another mechanism for generating assortment: an association between social interactions and horizontal transmission that creates a correlation between one's partner for interaction and the partner for transmission. This mechanism does not require repeated interactions, population structure, or individual recognition. We show that high levels of such interaction-transmission association greatly increase the potential for evolution of cooperation. With enough interaction-transmission association, cooperation can increase in frequency when initially rare even when there is horizontal transmission bias against it $\left(T_{A}<T_{B}\right)$.

356 How does non-vertical transmission generate assortment? Lewin-Epstein et al. [16] and LewinEpstein \& Hadany [17] suggested that microbes that induce their hosts to act altruistically can be favored by selection, which may help to explain the evolution of cooperation. From the kin selection point-of-view, if microbes can be transmitted horizontally from one host to another during host interactions, then following horizontal transmission the recipient host will carry microbes that are closely related to those of the donor host, even when the two hosts are (genetically) unrelated.

362 From the assortment point-of-view, infection by behavior-determining microbes during interactions effectively generates assortment because a recipient of help may be infected by a behavior-determining microbe and consequently become a helper. Cultural horizontal transmission can similarly generate assortment between cooperators and enhance the benefit of cooperation if cultural transmission and helping interactions occur between the same individuals, i.e. when there is interaction-transmission association, so that the recipient of help may also be the recipient of the cultural trait for cooperation. Thus, with horizontal transmission, "assortment between focal cooperative players and cooperative acts in their interaction environment" [9] is generated not because the helper is likely to be helped, but rather because the helped is likely to become a helper.

\section{Acknowledgements}

372 We thank Lilach Hadany, Ayelet Shavit, and Kaleda Krebs Denton for discussions and comments. This work was supported in part by the Clore Foundation Scholars Programme (OLE), the Morrison Institute for Population

374 and Resources Studies at Stanford University (MWF), Israel Science Foundation (YR 552/19), and Minerva Stiftung Center for Lab Evolution (YR). 


\section{Appendices}

\section{Appendix A Local stability criterion}

Let $f(p)=\lambda \cdot\left(p^{\prime}-p\right)$, where $\lambda>0$, and 0 and 1 are equilibria, that is, $f(0)=0$ and $f(1)=0$.

Set $p>p^{*}=0$. Using a linear approximation for $f(p)$ near 0 , we have

$$
p^{\prime}<p \Leftrightarrow f(p) / p<0 \Leftrightarrow \frac{f^{\prime}(0) \cdot p+O\left(p^{2}\right)}{p}<0 \Leftrightarrow f^{\prime}(0)+O(p)<0 .
$$

Therefore, by definition of big-O notation, if $f^{\prime}(0)<0$ then there exists $\epsilon>0$ such that for any local perturbation

$0<p<\epsilon$, it is guaranteed that $0<p^{\prime}<p$; that is, $p^{\prime}$ is closer to zero than $p$.

Set $p<p^{*}=1$ Using a linear approximation for $f(p)$ near 1 , we have

$$
1-p^{\prime}<1-p \Leftrightarrow-\frac{f(p)}{1-p}<0 \Leftrightarrow \frac{f^{\prime}(1)(p-1)+O\left((p-1)^{2}\right)}{p-1}<0 \Leftrightarrow f^{\prime}(1)-O(1-p)<0 .
$$

Therefore, if $f^{\prime}(1)<0$ then there exists $\epsilon>0$ such that for any $1-\epsilon<1-p<1$ we have $1-p^{\prime}<1-p$; that is, $p^{\prime}$ is closer to one than $p$.

\section{Appendix B Equilibria and stability}

Let $f(\hat{p})=\bar{w}\left(\hat{p}^{\prime}-\hat{p}\right)$. Then, using SymPy [22], a Python library for symbolic mathematics, this simplifies to

$$
f(\hat{p})=\bar{w}\left(\hat{p}^{\prime}-\hat{p}\right)=\beta_{1} \hat{p}^{3}+\beta_{2} \hat{p}^{2}+\beta_{3} \hat{p},
$$

where

$$
\begin{aligned}
& \beta_{1}=[c(1-v)-b(1-\alpha v)]\left(T_{A}-T_{B}\right), \\
& \beta_{2}=-\beta_{1}-\beta_{3}, \\
& \beta_{3}=\alpha b v T_{A}-c v\left(1-T_{B}\right)+\left(T_{A}-T_{B}\right) .
\end{aligned}
$$

If $T=T_{A}=T_{B}$ then $\beta_{1}=0$ and $\beta_{3}=-\beta_{2}=\alpha b v T-c v(1-T)$, and $f(\hat{p})$ becomes a quadratic polynomial,

$$
f(\hat{p})=\hat{p}(1-\hat{p})[\alpha b v T-c v(1-T)] .
$$

Clearly the only two equilibria are the fixations $\hat{p}=0$ and $\hat{p}=1$, which are are locally stable if $f^{\prime}(\hat{p})<0$ near

the equilibrium (see Appendix A), where $f^{\prime}(\hat{p})=(1-2 \hat{p})[\alpha b v T-c v(1-T)]$, so that

$$
\begin{aligned}
& f^{\prime}(0)=\alpha b v T-c v(1-T), \\
& f^{\prime}(1)=-\alpha b v T+c v(1-T) .
\end{aligned}
$$

In the general case where $T_{A} \neq T_{B}$, the coefficient $\beta_{1}$ is not necessarily zero, and $f(\hat{p})$ is a cubic polynomial. Therefore, three equilibria may exist, two of which are $\hat{p}=0$ and $\hat{p}=1$, and the third is

$$
\hat{p}^{*}=\frac{\beta_{3}}{\beta_{1}}=\frac{\alpha b v T_{A}-c v\left(1-T_{B}\right)+\left(T_{A}-T_{B}\right)}{[c(1-v)-b(1-\alpha v)]\left(T_{A}-T_{B}\right)} .
$$

Note that the sign of the cubic (Eq. B1) at positive (negative) infinity is equal (opposite) to the sign of $\beta_{1}$. If

$T_{A}>T_{B}$, then

$$
\beta_{1}<[c(1-\alpha v)-b(1-\alpha v)]\left(T_{A}-T_{B}\right)=(1-\alpha v)(c-b)\left(T_{A}-T_{B}\right)<0,
$$

since $c<b$ and $\alpha v<1$. Hence the signs of the cubic at positive and negative infinity are negative and positive, respectively. First, if $\beta_{3}<\beta_{1}$ then $1<\hat{p}^{*}$. Also, $f^{\prime}(0)<0$ and $f^{\prime}(1)>0$; that is, fixation of the defector 
phenotype $B$ is the only locally stable feasible equilibrium. Second, if $\beta_{1}<\beta_{3}<0$ then $0<\hat{p}^{*}<1$ and therefore $f^{\prime}(0)<0$ and $f^{\prime}(1)<0$ so that both fixations are locally stable and $\hat{p}^{*}$ separates the domains of attraction. Third, if $0<\beta_{3}$ then $\hat{p}^{*}<0$ and therefore $f^{\prime}(0)>0$ and $f^{\prime}(1)<0$; that is, fixation of the cooperator phenotype $A$ is the only locally stable legitimate equilibrium.

Similarly, if $T_{A}<T_{B}$, then

$$
\beta_{1}>[c(1-\alpha v)-b(1-\alpha v)]\left(T_{A}-T_{B}\right)=(1-\alpha v)(c-b)\left(T_{A}-T_{B}\right)>0,
$$

since $c<b$ and $\alpha v<1$, and the signs of the cubic at positive and negative infinity are positive and negative, respectively. First, if $\beta_{3}<0$ then $\hat{p}^{*}<0$ and therefore $f^{\prime}(0)<0$ and $f^{\prime}(1)>0$; that is, fixation of the defector phenotype $A$ is the only locally stable legitimate equilibrium. Second, if $0<\beta_{3}<\beta_{1}$ then $0<\hat{p}^{*}<1$ and therefore $f^{\prime}(0)>0$ and $f^{\prime}(1)>0$; that is, both fixations are locally unstable and $\hat{p}^{*}$ is a stable polymorphic equilibrium. Third, if $\beta_{1}<\beta_{3}$ then $\hat{p}^{*}>1$ and therefore $f^{\prime}(0)>0$ and $f^{\prime}(1)<0$, and fixation of the cooperator phenotype $A$ is the only locally stable feasible equilibrium.

This analysis can be summarized as follows:

1. Fixation of cooperation: if $(i) T=T_{A}=T_{B}$ and $c<b \cdot \frac{\alpha T}{1-T}$; or if $\left(\right.$ ii) $T_{A}>T_{B}$ and $0<\beta_{3}$; or if (iii) $T_{A}<T_{B}$ and $\beta_{1}<\beta_{3}$.

2. Fixation of the defection: if (iv) $T=T_{A}=T_{B}$ and $c>b \cdot \frac{\alpha T}{1-T}$; or if $(v) T_{A}>T_{B}$ and $\beta_{3}<\beta_{1}<0$; or if (vi) $T_{A}<T_{B}$ and $\beta_{3}<0$.

3. polymorphism of both phenotypes at $\hat{p}^{*}$ : if (vii) $T_{A}<T_{B}$ and $0<\beta_{3}<\beta_{1}$.

4. Fixation of either phenotype depending on initial frequency: if (viii) $T_{A}>T_{B}$ and $\beta_{1}<\beta_{3}<0$.

We now proceed to use the cost thresholds, $\gamma_{1}$ and $\gamma_{2}$, and the vertical transmission threshold, $\hat{v}$ (Eq. 13). First, assume $T_{A}<T_{B} . \beta_{3}<0$ requires $\gamma_{1}<c$. For $\beta_{3}<\beta_{1}$ we need $c\left[v\left(1-T_{B}\right)+(1-v)\left(T_{A}-T_{B}\right)\right]>$ $b v \alpha T_{B}+(1+b)\left(T_{A}-T_{B}\right)$. Note that the expression in the square brackets is positive if and only if $v>\hat{v}$. Thus, for $\beta_{3}<\beta_{1}$ we need $v>\hat{v}$ and $\gamma_{2}<c$ or $v<\hat{v}$ and $c<\gamma_{2}$, and for $0<\beta_{3}<\beta_{1}$ we need $v>\hat{v}$ and $\gamma_{2}<c<\gamma_{1}$, or $v<\hat{v}$ and $c<\min \left(\gamma_{1}, \gamma_{2}\right)$. For $\beta_{1}<\beta_{3}$ we need $v>\hat{v}$ and $c<\gamma_{2}$ or $v<\hat{v}$ and $\gamma_{2}<c$. However, some of these conditions cannot be met, since $v<\hat{v}$ implies $c<1<\gamma_{2}$.

Second, assume $T_{A}>T_{B} . \beta_{3}>0$ requires $\gamma_{1}>c$. For $\beta_{1}<\beta_{3}$ we need $c\left[v\left(1-T_{B}\right)+(1-v)\left(T_{A}-T_{B}\right)\right]<$ $b v \alpha T_{B}+(1+b)\left(T_{A}-T_{B}\right)$. Thus for $\beta_{1}<\beta_{3}$ we need $v>\hat{v}$ and $c<\gamma_{2}$ or $v<\hat{v}$ and $c>\gamma_{2}$. But $\hat{v}<0$ when $T_{A}>T_{B}$, and therefore we have $\beta_{1}<\beta_{3}$ if $c<\gamma_{2}$. Similarly, we have $\beta_{3}<\beta_{1}$ if $c>\hat{\gamma}_{2}$.

This analysis is summarized in Result 1.

\section{Appendix C Effect of interaction-transmission association on mean fitness}

To determine the effect of increasing $\alpha$ on the stable population mean fitness, $\bar{w}^{*}=1+(b-c) \hat{p}^{*}$, we must analyze its effect on $\hat{p}^{*}$,

$$
\frac{\partial \hat{p}^{*}}{\partial \alpha}=\frac{b T_{A}-c\left(1-T_{B}\right)+\left(T_{A}-T_{B}\right)}{b(1-\alpha)^{2}\left(T_{B}-T_{A}\right)} .
$$

Note that stable polymorphism implies $c<\gamma_{1}$, and because $\alpha<1$, we have

$$
c<\gamma_{1}=\frac{b \alpha T_{A}+\left(T_{A}-T_{B}\right)}{1-T_{B}}<\frac{b T_{A}+\left(T_{A}-T_{B}\right)}{1-T_{B}} .
$$

Therefore, the numerator in Eq. $\mathrm{C} 1$ is positive. Since $T_{A}<T_{B}$, the denominator in Eq. $\mathrm{C} 1$ is also positive, and hence the derivative $\partial \hat{p}^{*} / \partial \alpha$ is positive. Thus, the population mean fitness increases as interaction-transmission association $\alpha$ increases. 


\section{Appendix D Reduction principle}

446

448

We assume here that $v=1$, i.e. no oblique transmission, and therefore $\hat{p}=\tilde{p}$. Denote the frequencies of the pheno-genotypes $A M, B M, A m$, and $B m$ by $\tilde{\mathbf{p}}=\left(\tilde{p}_{1}, \tilde{p}_{2}, \tilde{p}_{3}, \tilde{p}_{4}\right)$. The frequencies of the pheno-genotypes in the next generation are defined by the recursion system,

$$
\begin{aligned}
\bar{w} \tilde{p}_{1}^{\prime}= & \tilde{p}_{1} x(1+b-c)\left(1-\left(1-\alpha_{1}\right)(1-x) T_{B}\right)+ \\
& \tilde{p}_{1}(1-x)(1-c)\left(1-\alpha_{1} T_{B} x-T_{B}(1-x)\right)+ \\
& \tilde{p}_{2} x(1+b) T_{A}\left(x+\alpha_{1}(1-x)\right)+ \\
& \tilde{p}_{2}(1-x) x\left(1-\alpha_{1}\right) T_{A}, \\
\bar{w} \tilde{p}_{2}^{\prime}= & \tilde{p}_{1} x(1+b-c)\left(1-\alpha_{1}\right)(1-x) T_{B}+ \\
& \tilde{p}_{1}(1-x)(1-c)\left(\alpha_{1} T_{B}+\left(1-\alpha_{1}\right)(1-x) T_{B}\right)+ \\
& \tilde{p}_{2} x(1+b)\left(1-\alpha_{1} T_{A}(1-x)-T_{A} x\right)+ \\
& \tilde{p}_{2}(1-x)\left(1-\left(1-\alpha_{1}\right) x T_{A}\right), \\
\bar{w} \tilde{p}_{3}^{\prime}= & \tilde{p}_{3} x(1+b-c)\left(1-\left(1-\alpha_{2}\right)(1-x) T_{B}\right)+ \\
& \tilde{p}_{3}(1-x)(1-c)\left(1-\alpha_{2} T_{B} x-T_{B}(1-x)\right)+ \\
& \tilde{p}_{4} x(1+b) T_{A}\left(x+\alpha_{2}(1-x)\right)+ \\
& \tilde{p}_{4}(1-x) x\left(1-\alpha_{2}\right) T_{A}, \\
\bar{w} \tilde{p}_{4}^{\prime}= & \tilde{p}_{3} x(1+b-c)\left(1-\alpha_{2}\right)(1-x) T_{B}+ \\
& \tilde{p}_{3}(1-x)(1-c)\left(\alpha_{2} T_{B}+\left(1-\alpha_{2}\right)(1-x) T_{B}\right)+ \\
& \tilde{p}_{4} x(1+b)\left(1-\alpha_{2} T_{A}(1-x)-T_{A} x\right)+ \\
& \tilde{p}_{4}(1-x)\left(1-\left(1-\alpha_{2}\right) x T_{A}\right),
\end{aligned}
$$

450

452

462

454

where $x=\tilde{p}_{1}+\tilde{p}_{3}$ is the total frequency of the cooperative phenotype $A$, and $\bar{w}=1+(b-c) x$ is the population mean fitness.

The equilibrium where only allele $M$ is present is $\tilde{\mathbf{p}}^{*}=\left(\tilde{p}^{*}, 1-\tilde{p}^{*}, 0,0\right)$, where

$$
\tilde{p}^{*}=\frac{c\left(1-T_{B}\right)-b \alpha_{1} T_{A}-\left(T_{A}-T_{B}\right)}{b\left(1-\alpha_{1}\right)\left(T_{A}-T_{B}\right)},
$$

setting $\alpha=\alpha_{1}$ and $v=1$ in Eq. 12. When $v=1, \tilde{p}^{*}$ is a feasible polymorphism $\left(0<\tilde{p}^{*}<1\right)$ if $T_{A}<T_{B}$ and $\gamma_{2}<c<\gamma_{1}$ (Result 1).

The local stability of $\tilde{\mathbf{p}}^{*}$ to the introduction of allele $m$ is determined by the linear approximation $\mathbf{L}^{*}$ of the transformation in Eq. D1 near $\tilde{\mathbf{p}}^{*}$ (i.e., the Jacobian of the transformation at the equilibrium). $\mathbf{L}^{*}$ is known to have a block structure, with the diagonal blocks occupied by the matrices $\mathbf{L}_{i n}^{*}$ and $\mathbf{L}_{e x}^{*}[1,20]$. The latter is the external stability matrix: the linear approximation to the transformation near $\tilde{\mathbf{p}}^{*}$ involving only the pheno-genotypes $A m$ and $B m$, derived from Eq. D1, with $\bar{w}^{*}=1+(b-c) \tilde{p}^{*}$ as the stable population mean fitness,

$$
\begin{aligned}
& \mathbf{L}_{e x}^{*}=\frac{1}{\bar{w}^{*}}\left[\begin{array}{ll}
l_{11} & l_{12} \\
l_{21} & l_{22}
\end{array}\right]=\frac{1}{\bar{w}^{*}}\left[\begin{array}{cc}
\frac{\partial \bar{w} \tilde{p}_{3}^{\prime}}{\partial \tilde{p}_{3}}\left(\tilde{\mathbf{p}}^{*}\right) & \frac{\partial \bar{w} \tilde{p}_{3}^{\prime}}{\partial \tilde{p}_{4}}\left(\tilde{\mathbf{p}}^{*}\right) \\
\frac{\partial \bar{w} \tilde{\tilde{p}}_{4}^{\prime}}{\partial \tilde{p}_{3}}\left(\tilde{\mathbf{p}}^{*}\right) & \frac{\partial \bar{w} \tilde{p}_{4}^{\prime}}{\partial \tilde{p}_{4}}\left(\tilde{\mathbf{p}}^{*}\right)
\end{array}\right]= \\
& \frac{1}{\bar{w}^{*}}\left[\begin{array}{cc}
\left(1+b \tilde{p}^{*}-c\right)\left(1-T_{B}\left(1-\tilde{p}^{*}\right)\right)+b \tilde{p}^{*} \alpha_{2} T_{B}\left(1-\tilde{p}^{*}\right) & \left(1+b \tilde{p}^{*}\right) T_{A} \tilde{p}^{*}+b \tilde{p}^{*} \alpha_{2} T_{A}\left(1-\tilde{p}^{*}\right) \\
\left(1+b \tilde{p}^{*}-c\right) T_{B}\left(1-\tilde{p}^{*}\right)-b \tilde{p}^{*} \alpha_{2} T_{B}\left(1-\tilde{p}^{*}\right) & \left(1+b \tilde{p}^{*}\right)\left(1-T_{A} \tilde{p}^{*}\right)-b \tilde{p}^{*} \alpha_{2} T_{A}\left(1-\tilde{p}^{*}\right)
\end{array}\right] .
\end{aligned}
$$

Because we assume that $\tilde{\mathbf{p}}^{*}$ is internally stable (i.e. locally stable to small perturbations in the frequencies

of $A M$ and $B M$ ), the stability of $\tilde{\mathbf{p}}^{*}$ is determined by the eigenvalues of the external stability matrix $\mathbf{L}_{e x}^{*}$. This is a positive matrix, and due to the Perron-Frobenius theorem, the leading eigenvalue of $\mathbf{L}_{e x}^{*}$ is real and positive. Thus, if the leading eigenvalue is less (greater) than one, then the equilibrium $\tilde{\mathbf{p}}^{*}$ is externally stable (unstable) and allele $m$ cannot (can) invade the population of allele $M$. The eigenvalues of $\mathbf{L}_{e x}^{*}$ are the roots 
since $c>0,0<\tilde{p}^{*}<1$. That is,

$$
\begin{aligned}
& \operatorname{sign} R(1)=\operatorname{sign} \operatorname{det}\left[\begin{array}{cc}
-c\left(1-\tilde{p}^{*}\right) & c \tilde{p}^{*} \\
\left(1-\tilde{p}^{*}\right)\left[\left(1+b \tilde{p}^{*}-c\right) T_{B}-b \alpha_{2} T_{B} \tilde{p}^{*}\right] & \tilde{p}^{*}\left[-\left(1+b \tilde{p}^{*}\right) T_{A}-b \alpha_{2} T_{A}\left(1-\tilde{p}^{*}\right)+c\right]
\end{array}\right]= \\
& \operatorname{sign}\left[c \tilde{p}^{*}\left(1-\tilde{p}^{*}\right) \cdot \operatorname{det}\left[\begin{array}{cc}
-1 & 1 \\
\left(1+b \tilde{p}^{*}-c\right) T_{B}-b \alpha_{2} T_{B} \tilde{p}^{*} & -\left(1+b \tilde{p}^{*}\right) T_{A}-b \alpha_{2} T_{A}\left(1-\tilde{p}^{*}\right)+c
\end{array}\right]\right]= \\
& \text { sign det }\left[\begin{array}{cc}
-1 & 1 \\
\left(1+b \tilde{p}^{*}-c\right) T_{B}-b \alpha_{2} T_{B} \tilde{p}^{*} & -\left(1+b \tilde{p}^{*}\right) T_{A}-b \alpha_{2} T_{A}\left(1-\tilde{p}^{*}\right)+c
\end{array}\right],
\end{aligned}
$$

Substituting $\tilde{p}^{*}$ from Eq. D2, we get

$$
\begin{aligned}
R(1)<0 \Leftrightarrow & {\left[c\left(1-T_{B}\right)-b \alpha_{1} T_{A}-\left(T_{A}-T_{B}\right)\right] \frac{1-\alpha_{2}}{1-\alpha_{1}}-c\left(1-T_{B}\right)+b \alpha_{2} T_{A}+\left(T_{A}-T_{B}\right)<0 \Leftrightarrow } \\
& \left(1-\alpha_{2}\right)\left[c\left(1-T_{B}\right)-b \alpha_{1} T_{A}-\left(T_{A}-T_{B}\right)\right]<\left(1-\alpha_{1}\right)\left[c\left(1-T_{B}\right)-b \alpha_{2} T_{A}-\left(T_{A}-T_{B}\right)\right] \Leftrightarrow \\
& -b \alpha_{1} T_{A}-\alpha_{2} c\left(1-T_{B}\right)+\alpha_{2}\left(T_{A}-T_{B}\right)<-b \alpha_{2} T_{A}-\alpha_{1} c\left(1-T_{B}\right)+\alpha_{1}\left(T_{A}-T_{B}\right) \Leftrightarrow \\
& \alpha_{1}\left[c\left(1-T_{B}\right)-b T_{A}-\left(T_{A}-T_{B}\right)\right]<\alpha_{2}\left[c\left(1-T_{B}\right)-b T_{A}-\left(T_{A}-T_{B}\right)\right] \Leftrightarrow \\
& \alpha_{1}\left[b T_{A}+\left(T_{A}-T_{B}\right)-c\left(1-T_{B}\right)\right]>\alpha_{2}\left[b T_{A}+\left(T_{A}-T_{B}\right)-c\left(1-T_{B}\right)\right] .
\end{aligned}
$$

We assumed $c<\gamma_{1}$, and since $0 \leq \alpha_{1} \leq 1$,

$$
\begin{aligned}
& c<\gamma_{1}=\frac{b \alpha_{1} T_{A}+\left(T_{A}-T_{B}\right)}{1-T_{B}} \Leftrightarrow \\
& 0<b \alpha_{1} T_{A}+\left(T_{A}-T_{B}\right)-c\left(1-T_{B}\right) \Rightarrow \\
& 0<b T_{A}+\left(T_{A}-T_{B}\right)-c\left(1-T_{B}\right) .
\end{aligned}
$$

Combining inequalities D7 and D8, we find that $R(1)<0$ if and only if $\alpha_{1}>\alpha_{2}$, which is a sufficient condition $m$, is less than $\alpha_{1}$, the interaction-transmission association of the resident allele $M$, then invasion will be successful.

Determining a necessary and sufficient condition for successful invasion is more complicated, requiring analysis of the sign of $R^{\prime}(1)$. However, we have numerically validated that the leading eigenvalue is greater than one if and only if $\alpha_{1}>\alpha_{2}$. 
490

492

494

\section{References}

[1] Altenberg, L., Liberman, U. \& Feldman, M. W. 2017 Unified reduction principle for the evolution of mutation, migration, and recombination. Proc. Natl. Acad. Sci. U. S. A., 114(12), E2392-E2400. (doi: 10.1073/pnas.1619655114)

[2] Axelrod, R. \& Hamilton, W. D. 1981 The evolution of cooperation. Science, 211(4489), 1390-1396.

[3] Bijma, P. \& Aanen, D. K. 2010 Assortment, Hamilton's rule and multilevel selection. Proc. R. Soc. B Biol. Sci., 277(1682), 673-675. (doi:10.1098/rspb.2009.1093)

[4] Cavalli-Sforza, L. L. \& Feldman, M. W. 1981 Cultural transmission and evolution: A quantitative approach. 16. Princeton University Press.

[5] Dugatkin, L. A. 1997 Cooperation among animals: An evolutionary perspective. Oxford University Press on Demand.

[6] Eshel, I. \& Cavalli-Sforza, L. L. 1982 Assortment of encounters and evolution of cooperativeness. Proceedings of the National Academy of Sciences, 79(4), 1331-1335.

[7] Feldman, M. W. 1972 Selection for linkage modification: I. Random mating populations. Theor. Popul. Biol., 3, 324-346.

[8] Feldman, M. W., Cavalli-Sforza, L. L. \& Peck, J. R. 1985 Gene-culture coevolution: models for the evolution of altruism with cultural transmission. Proceedings of the National Academy of Sciences, 82(17), 5814-5818.

[9] Fletcher, J. A. \& Doebeli, M. 2009 A simple and general explanation for the evolution of altruism. Proc. R. Soc. B Biol. Sci., 276(1654), 13-19. (doi:10.1098/rspb.2008.0829)

[10] Gurevich, Y., Lewin-Epstein, O. \& Hadany, L. 2020 The evolution of paternal care: a role for microbes? Philos. Trans. R. Soc. B Biol. Sci., 375(1808), 20190 599. (doi:10.1098/rstb.2019.0599)

[11] Haldane, J. B. S. 1932 The Causes of Evolution. London: Longmans.

[12] Hamilton, W. D. 1964 The genetical evolution of social behaviour. ii. Journal of Theoretical Biology, 7(1), 17-52.

[13] Jaeggi, A. V. \& Gurven, M. 2013 Natural cooperators: food sharing in humans and other primates. Evolutionary Anthropology: Issues, News, and Reviews, 22(4), 186-195.

[14] Karlin, S., Lieberman, U. \& Liberman, U. 1975 Random temporal variation in selection intensities: One-locus two-allele model. J. Math. Biol., 6(3), 1-17. (doi:10.1016/0040-5809(74)90016-1)

[15] Krams, I., Krama, T., Igaune, K. \& Mänd, R. 2008 Experimental evidence of reciprocal altruism in the pied flycatcher. Behavioral Ecology and Sociobiology, 62(4), 599-605.

[16] Lewin-Epstein, O., Aharonov, R. \& Hadany, L. 2017 Microbes can help explain the evolution of host altruism. Nature Communications, 8, 14040.

[17] Lewin-Epstein, O. \& Hadany, L. 2020 Host-microbiome coevolution can promote cooperation in a rockpaper-scissors dynamics. Proc. R. Soc. B Biol. Sci., 287(1920), 20192 754. (doi:10.1098/rspb.2019.2754)

[18] Liberman, U. 1988 External stability and ESS: criteria for initial increase of new mutant allele. J. Math. Biol., 26(4), 477-485. (doi:10.1007/BF00276375)

[19] Liberman, U. \& Feldman, M. W. 1986 A general reduction principle for genetic modifiers of recombination. Theor. Popul. Biol., 30(3), 341-71.

[20] Liberman, U. \& Feldman, M. W. 1986 Modifiers of mutation rate: A general reduction principle. Theor. Popul. Biol., 30, 125-142. 
bioRxiv preprint doi: https://doi org/10.1101/2020.12 08 416560; this version posted December 9,2020 . The copyright holder for this preprint (which was not certified by peer review) is the author/funder, who has granted bioRxiv a license to display the preprint in perpetuity. It is made available under aCC-BY-NC 4.0 International license.

[21] Lycett, S. J. \& Gowlett, J. A. 2008 On questions surrounding the acheulean 'tradition'. World Archaeology, 40(3), 295-315.

[22] Meurer, A., Smith, C. P., Paprocki, M., Čertík, O., Kirpichev, S. B., Rocklin, M., Kumar, A., Ivanov, S., Moore, J. K. et al. 2017 Sympy: symbolic computing in python. PeerJ Computer Science, 3, e103.

[23] Ram, Y., Liberman, U. \& Feldman, M. W. 2018 Evolution of vertical and oblique transmission under fluctuating selection. Proceedings of the National Academy of Sciences, 115(6), E1174-E1183.

[24] Rice, G. E. \& Gainer, P. 1962 "Altruism" in the albino rat. Journal of Comparative and Physiological Psychology, 55(1), 123.

[25] Richerson, P. J. \& Boyd, R. 2008 Not by genes alone: How culture transformed human evolution. University of Chicago Press.

[26] Sinervo, B., Chaine, A., Clobert, J., Calsbeek, R., Hazard, L., Lancaster, L., McAdam, A. G., Alonzo, S., Corrigan, G. et al. 2006 Self-recognition, color signals, and cycles of greenbeard mutualism and altruism. Proceedings of the National Academy of Sciences, 103(19), 7372-7377.

544 [27] Stacey, P. B. \& Koenig, W. D. (eds) 1990 Cooperative breeding in birds: long term studies of ecology and behaviour. Cambridge University Press.

546 [28] Woodcock, S. 2006 The significance of non-vertical transmission of phenotype for the evolution of altruism. Biology and Philosophy, 21(2), 213-234.

548 [29] Zefferman, M. R. 2016 Mothers teach daughters because daughters teach granddaughters: the evolution of sex-biased transmission. Behav. Ecol., 27(4), 1172-1181. (doi:10.1093/beheco/arw022) 
Table 1: Interaction frequency, fitness, and transmission probabilities.

\begin{tabular}{llllll}
\hline Phenotype $\phi_{1}$ & Phenotype $\phi_{2}$ & Frequency & Fitness of $\phi_{1}$ & \multicolumn{2}{l}{$P\left(\phi_{1}=A\right)$ via horizontal transmission: } \\
\cline { 5 - 6 } & & & from partner, $\alpha$ & from population, $(1-\alpha)$ \\
\hline$A$ & $A$ & $\hat{p}^{2}$ & $1+b-c$ & 1 & $\hat{p}+(1-\hat{p})\left(1-T_{B}\right)$ \\
$A$ & $B$ & $\hat{p}(1-\hat{p})$ & $1-c$ & $1-T_{B}$ & $\hat{p}+(1-\hat{p})\left(1-T_{B}\right)$ \\
$B$ & $A$ & $\hat{p}(1-\hat{p})$ & $1+b$ & $T_{A}$ & $\hat{p} T_{A}$ \\
$B$ & $B$ & $(1-\hat{p})^{2}$ & 1 & 0 & $\hat{p} T_{A}$ \\
\hline
\end{tabular}

Table 2: Model variables and parameters.

\begin{tabular}{lll}
\hline Symbol & Description & Values \\
\hline$A$ & Cooperator phenotype & \\
$B$ & Defector phenotype & \\
$p$ & Frequency of phenotype $A$ among adults & {$[0,1]$} \\
$\tilde{p}$ & Frequency of phenotype $A$ among parents & {$[0,1]$} \\
$\hat{p}$ & Frequency of phenotype $A$ among juveniles & {$[0,1]$} \\
$v$ & Vertical transmission rate & {$[0,1]$} \\
$c$ & Cost of cooperation & $(0,1)$ \\
$b$ & Benefit of cooperation & $c<b$ \\
$\alpha$ & Probability of interaction-transmission association & {$[0,1]$} \\
$T_{A}, T_{B}$ & Horizontal transmission rates of phenotype $A$ and $B$ & $(0,1)$ \\
& & \\
\hline
\end{tabular}




\section{Figures}

a
\begin{tabular}{|l|c|c|}
\hline & $\begin{array}{c}\boldsymbol{\phi}_{\mathbf{2}}=A \\
\text { cooperator }\end{array}$ & $\begin{array}{c}\boldsymbol{\phi}_{2}=B \\
\text { defector }\end{array}$ \\
\hline $\begin{array}{c}\boldsymbol{\phi}_{\mathbf{1}}=A \\
\text { cooperator }\end{array}$ & $1+b-c$ & $1-c$ \\
\hline $\begin{array}{c}\boldsymbol{\phi}_{\mathbf{1}}=B \\
\text { defector }\end{array}$ & $1+b$ & 1 \\
\hline
\end{tabular}

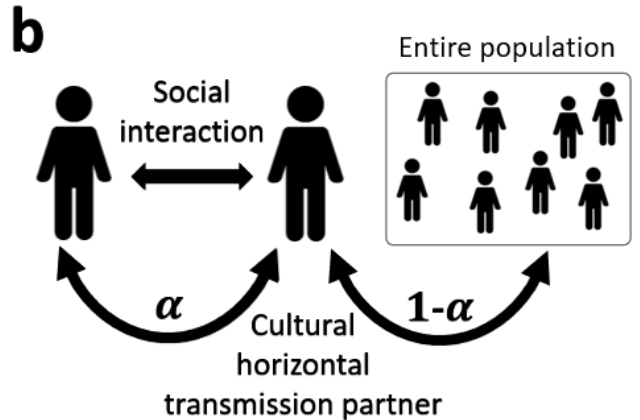

C

d
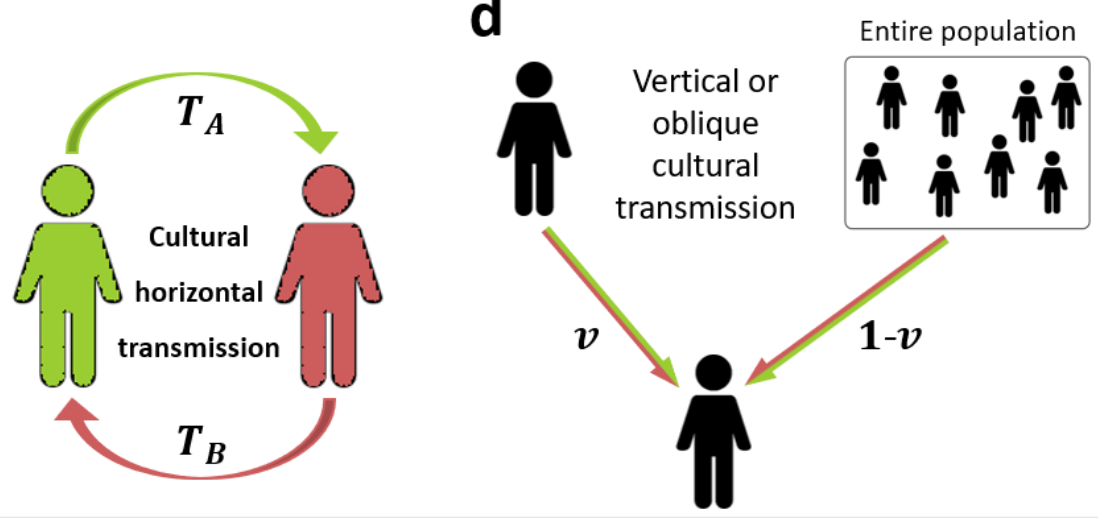

Figure 1: Model illustration. (a) Prisoners' dilemma payoff matrix, showing The fitness of phenotype $\phi_{1}$ when interacting with phenotype $\phi_{2}$. (b) Individuals socially interact in pairs in a prisoners' dilemma game. Horizontal cultural transmission occurs from a random individual in the population, with probability $1-\alpha$, or from the social partner, with probability $\alpha$, where $\alpha$ is the interactiontransmission association parameter. (c) The probabilities of successful horizontal cultural transmission of phenotypes $A$ and $B$ are $T_{A}$ and $T_{B}$, respectively. (d) Offspring inherit their parent's phenotype with probability $v$, or the phenotype of a random non-parental adult with probability $1-v$. 

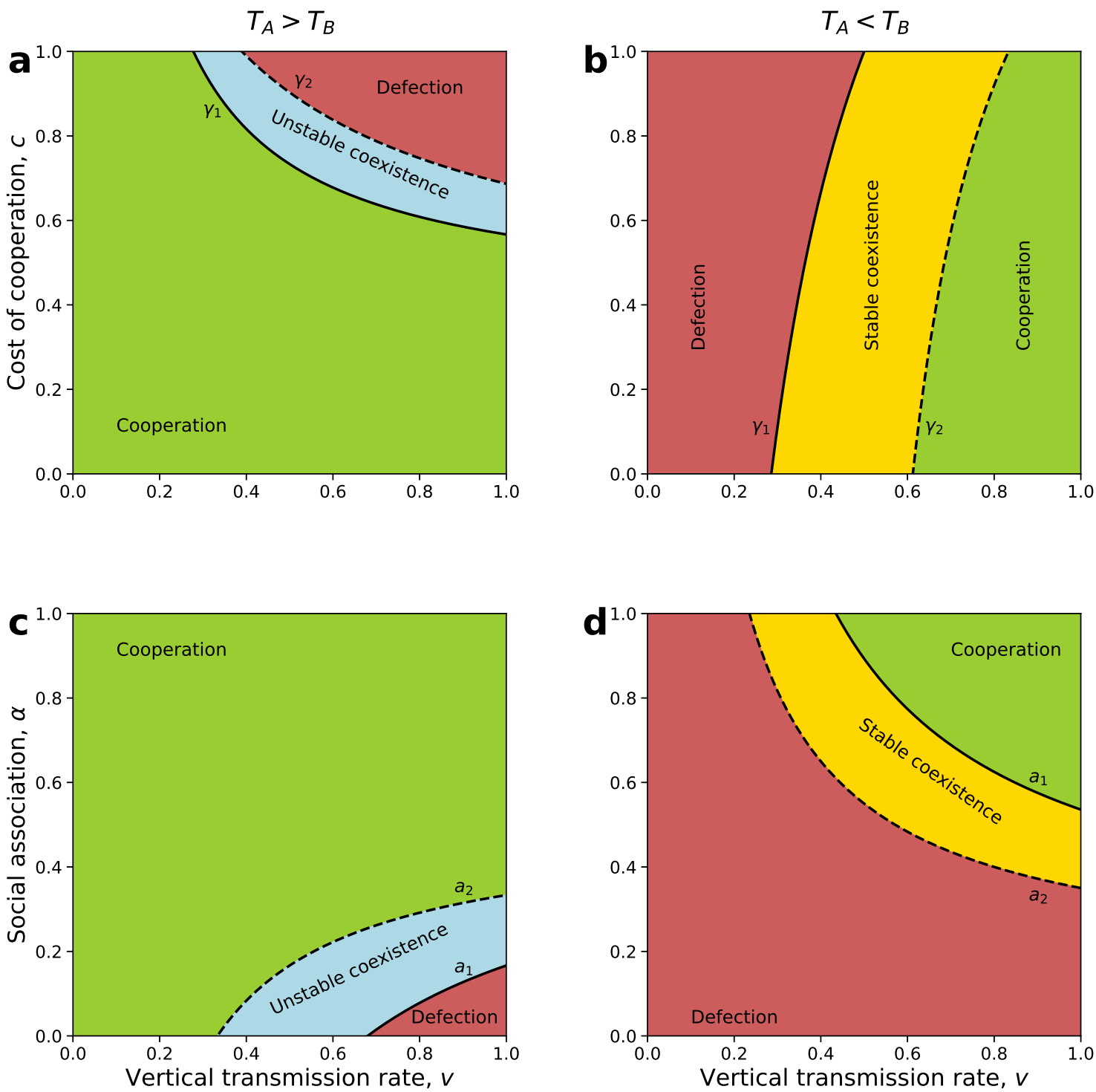

Figure 2: Evolution of cooperation under vertical, oblique, and horizontal cultural transmission. The figure shows parameter ranges for global fixation of cooperation (green), global fixation of defection (red), fixation of either cooperation or defection depending on the initial conditions, i.e. unstable polymorphism (blue), and stable polymorphism of cooperation and defection (yellow). In all cases the vertical transmission rate $v$ is on the x-axis. (a-b) Cost of cooperation $c$ is on the $y$-axis and the cost thresholds $\gamma_{1}$ and $\gamma_{2}$ (Eqs. 13) are represented by the solid and dashed lines, respectively. (cd) Interaction-transmission association $\alpha$ is on the y-axis and the interaction-transmission association thresholds $a_{1}$ and $a_{2}$ (Eqs. 18) are represented by the solid and dashed lines, respectively. Horizontal transmission is biased in favor of cooperation, $T_{A}>T_{B}$, in (a) and (c), or defection, $T_{A}<T_{B}$, in (b) and (d). Here, $T_{A}=0.5$, and (a) $b=1.2, T_{B}=0.4, \alpha=0.4 ;(\mathbf{b}) b=2, T_{B}=0.7, \alpha=0.7$; (c) $b=1.2, T_{B}=0.4, c=0.5 ;(\mathbf{d}) b=2, T_{B}=0.7, c=0.5$. 

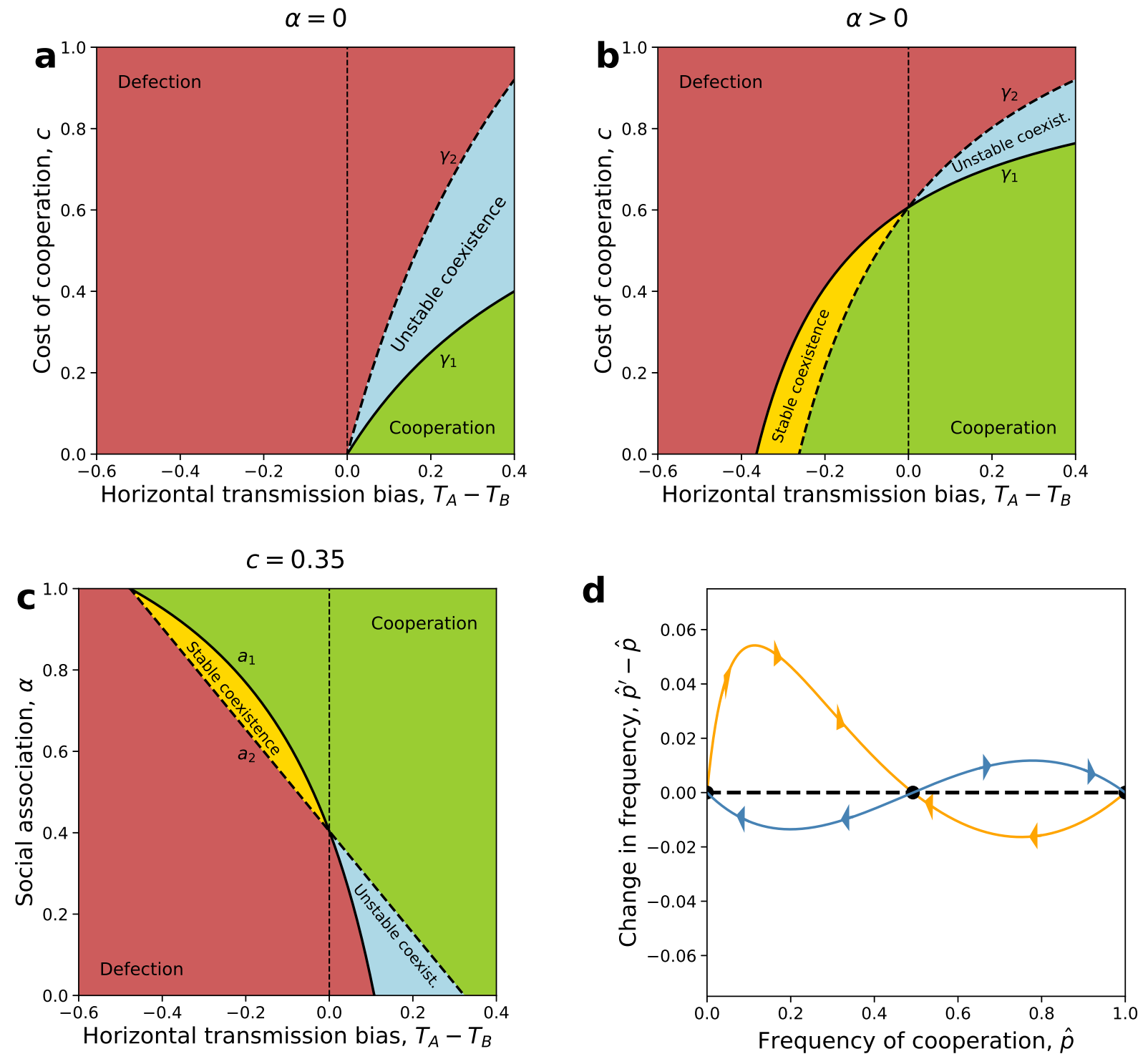

Figure 3: Evolution of cooperation under vertical and horizontal cultural transmission ( $v=1)$. The figure shows parameter ranges for global fixation of cooperation (green), global fixation of defection (red), fixation of either cooperation or defection depending on the initial conditions, i.e. unstable polymorphism (blue), and stable polymorphism of cooperation and defection (yellow). (ac) The horizontal transmission bias $\left(T_{A}-T_{B}\right)$ is on the x-axis. In panels (a) and (b), the cost of cooperation $c$ is on the y-axis and the cost thresholds $\gamma_{1}$ and $\gamma_{2}$ (Eq. 13) are the solid and dashed lines, respectively. In panel (c), interaction-transmission association $\alpha$ is on the y-axis and the interactiontransmission association thresholds $a_{1}$ and $a_{2}$ (Eqs. 18) are the solid and dashed lines, respectively. Here, $b=1.3, T_{A}=0.4, v=1$, (a) $\alpha=0$, (b) $\alpha=0.7$, (c) $c=0.35$. (d) Change in frequency of cooperation among juveniles $\left(\hat{p}^{\prime}-\hat{p}\right)$ as a function of the frequency $(\hat{p})$, see Eq. 11 . The orange curve shows convergence to a stable polymorphism $\left(T_{A}=0.4, T_{B}=0.9, b=12, c=0.35, v=1\right.$, and $\alpha=0.45)$. The blue curve shows fixation of either cooperation or defection, depending on the initial frequency $\left(T_{A}=0.5, T_{B}=0.1, b=1.3, c=0.904, v=1\right.$, and $\left.\alpha=0.4\right)$. Black circles show the three equilibria. 

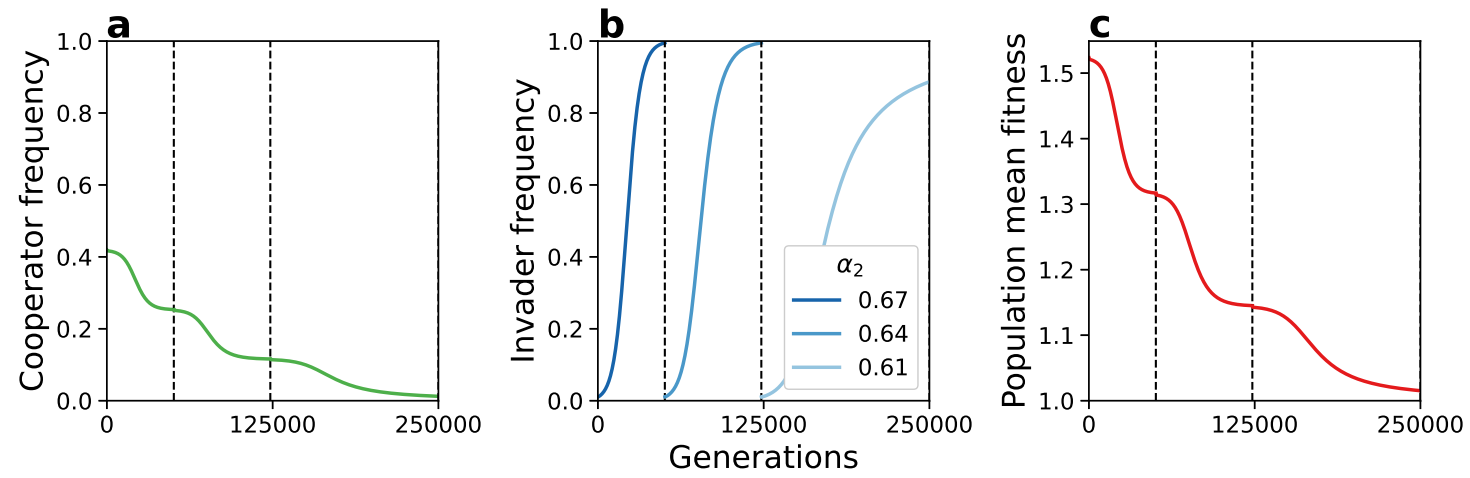

Figure 4: Reduction principle for interaction-transmission association. Consecutive fixation of modifier alleles that reduce interaction-transmission association $\alpha$ in numerical simulations of evolution with two modifier alleles (Eq. D1). When an invading modifier allele is established in the population (frequency $>99.95 \%$ ), a new modifier allele that reduces interaction-transmission association by $5 \%$ is introduced (at initial frequency $0.5 \%$ ). (a) The frequency of the cooperative phenotype $A$ over time. (b) The frequency of the invading modifier allele $m$ over time. (c) The population mean fitness $(\bar{w})$ over time. Here, $c=0.05, b=1.3, T_{A}=0.4<T_{B}=0.7$, initial interaction-transmission association $\alpha_{1}=0.7$, lower interaction-transmission association threshold $a_{2}=0.605$.
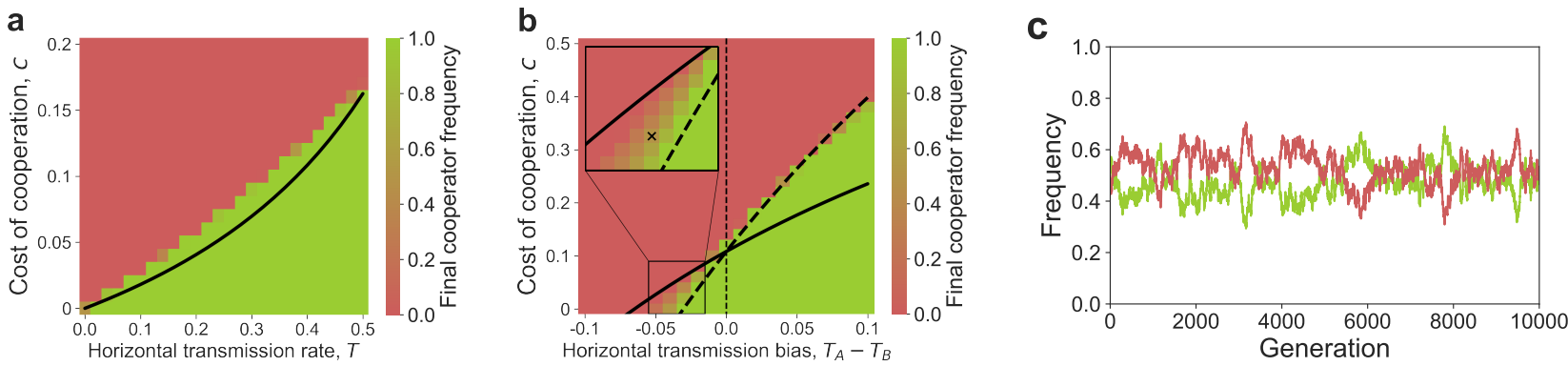

Figure 5: Evolution of cooperation in a structured population. (a-b) The expected frequency of cooperators in a structured population after 10,000 generations is shown (red for 0\%, green for 100\%) as a function of both the cost of cooperation, $c$, on the y-axis, and either the symmetric horizontal transmission rate, $T=T_{A}=T_{B}$, on the x-axis of panel (a), or the transmission bias, $T_{A}-T_{B}$, on the $\mathrm{x}$-axis of panel (b). Black curves represent the cost thresholds for the evolution of cooperation in a well-mixed population with interaction-transmission association, where $\alpha=1 / 8$ in inequality 15 for panel (a) and in Eqs. 13 for panel (b). The inset in panel (b) focuses on an area of the parameter range in which neither phenotype is fixed throughout the simulation, maintaining a stochastic locally stable polymorphism [14]. This stochastic polymorphism is illustrated in panel (c), which shows the frequency of cooperators (green) and defectors (red) over time for the parameter set marked by an $x$ in panel (b). In all cases, the population evolves on a 100-by-100 grid. Cooperation and horizontal transmission are both local between neighboring sites, and each site has 8 neighbors. Selection operates globally (see Figure S1 for results from a model with local selection). Simulations were stopped at generation 10,000 or if one of the phenotypes fixed. 50 simulations were executed for each parameter set. Benefit of cooperation, $b=1.3$; perfect vertical transmission $v=1$. (a) Symmetric horizontal transmission, $T=T_{A}=T_{B}$; (b) Horizontal transmission rate $T_{A}$ is fixed at 0.4 , and $T_{B}$ varies, $0.3<T_{B}<0.5$. (c) Horizontal transmission rates $T_{A}=0.4<T_{B}=0.435$ and cost of cooperation $c=0.02$. 
a

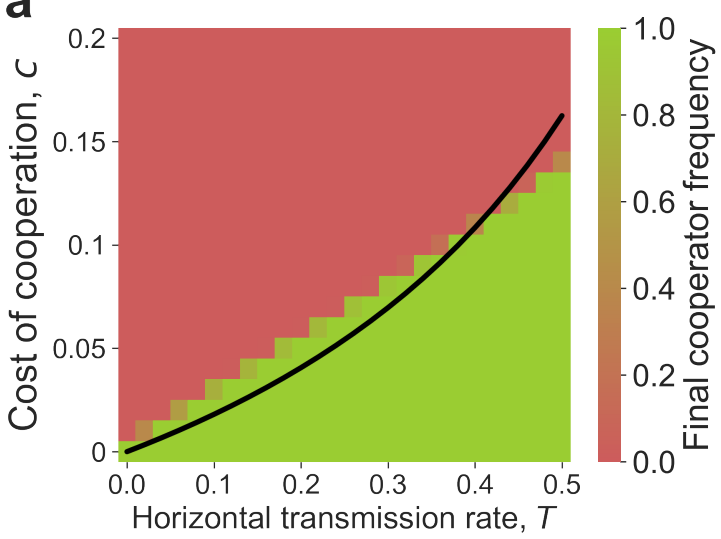

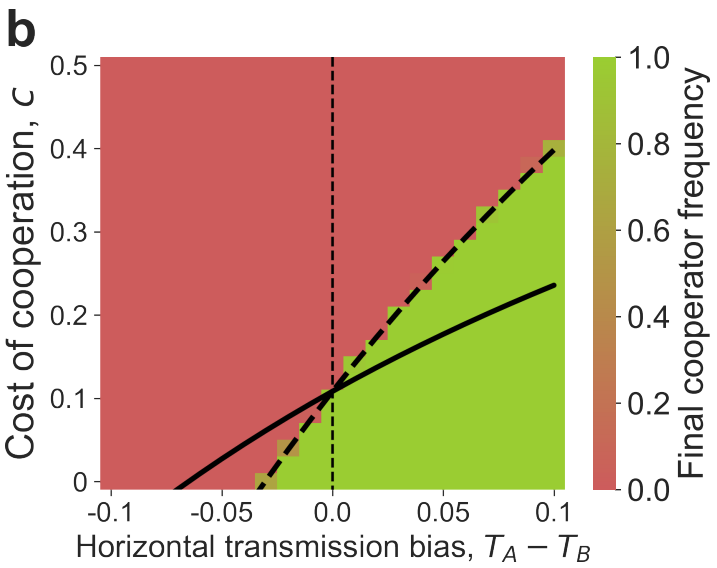

Figure S1: Evolution of cooperation in a structured population with local selection. The expected frequency of cooperators in a structured population after 10,000 generations is shown (red for $0 \%$, green for $100 \%$ ) as a function of both the cost of cooperation $(c)$ on the y-axis, and the symmetric horizontal transmission rate $\left(T=T_{A}=T_{B}\right)$ on the x-axis of panel (a), or the transmission bias $T_{A}-T_{B}$ on the $\mathrm{x}$-axis of panel (b). Cooperation and horizontal transmission are both local between neighboring sites, and each site had 8 neighbors. Selection operates locally (see Figure 5 for results from a model with global selection). The black curves represent the cost thresholds for the evolution of cooperation in a well-mixed population with interaction-transmission association, where $\alpha=1 / 8$ in inequality 15 for panel (a) and in Eqs. 13 for panel (b). The population evolves on a 100-by-100 grid. Simulations were stopped at generation 10,000 or if one of the phenotypes fixed. 50 simulations were executed for each parameter set. Here, benefit of cooperation, $b=1.3$; perfect vertical transmission $v=1$. (a) Symmetric horizontal transmission, $T=T_{A}=T_{B}$. (b) Horizontal transmission rate $T_{A}$ is fixed at 0.4 , and $T_{B}$ varies, $0.3<T_{B}<0.5$. 